\title{
EVALUATION OF THE ROLE OF THE ACCENT METHOD OF VOICE THERAPY AFTER MICROLARYNGEAL PHONOSURGERY
}

\author{
Samia El-Sayed Bassiouny*, Alia Mahmoud El-Shobary*, Nirvana Gamal El-Dien \\ Hafez*, Ayman Mohamed Shawky**and Amr Sayed Mousa**
}

*Unit of Phoniatrics, Faculty of
Medicine, Ain-Shams
University. **Unit of
Phoniatrics, Medical Military
Academy.
Corresponding :
Amr Sayed Mohamed Mousa
Mobile201002836877
E mail:
amr_saymousa@yahoo.com
Received: 25/7/2019
Accepted: 29/8/2019

\begin{abstract}
:
Background: The accent method (AM) is one of the holistic approaches for behavior readjustment technique (BRAT). Anatomic physiologic explanation for the accent method is the rhythmic pairing of timing with respiration and phonation may promote motor learning of new phonatory behaviors that will enhance the Bernoulli's effect to restore the glottic wave with better adjustment and symmetry of the vibrator (vocal fold mucosa).
\end{abstract}

Aim of the work: To evaluate the role of applying the accent method of voice therapy on patients with different benign vocal fold lesions post-operatively in order to emphasize the importance of postoperative Accent Method in improving the vocal performance.

Patients and Methods: This study was conducted on 100 patients: 50 adult patients of both sexes received "accent exercises" of voice therapy 1week after microlaryngeal phonosurgery which was the study group (G1) and other 50 adult patients of both sexes as a control group (G2) received only voice hygienic advice. Three assessments were done, the first was before the microlaryngeal phonosurgery, the second was one week after surgery and the third was after the course of the voice therapy which compared subjective and objective voice evaluation parameters investigating the therapeutic effect of voice therapy.

Results: For the second assessment (after microlaryngeal phonosurgery), there were no statistically significant differences between the 2 groups in all voice evaluation parameters. For the third assessment (after the "accent exercises" given to G1 only and voice hygienic advice given to both groups), there were statistically significant/highly significant differences in favor of G1 as regard auditory perceptual assessment (APA), high-speed video-kymography parameters, acoustic analysis and aerodynamic measures.

Conclusion: Since the minimal associated pathological lesions (MAPLs) represent $10 \%$ of dysphonia in vocology which likely a result of phonotrauma (abuse and misuse of voice), voice therapy after doing microlaryngeal phonosurgery is almost always a good idea in order to make the person aware of circumstances and habits of voice use that may lead to recurrence of the lesion in the first place.

Keywords: microlaryngeal phonosurgery, accent method (AM), minimal associated pathological lesions (MAPLs), dysphonia, the vibrator (vocal fold mucosa).

\section{INTRODUCTION:}

Dysphonia is a general term for voice disorders as the main symptom of laryngeal lesions; it seriously affects the life quality of patients and interpersonal communication. It is known that the primary factor for 
dysphonia is imperfect closure of the glottis which is easy to diagnose by laryngoscopy. Also, vocal fold stiffness or alteration of it's layered structure is another factor responsible for dysphonia ${ }^{(1)}$.

Treatment is mainly comprehensive therapy either conservative treatment or surgical interventions. Conservative treatments are mainly based on voice hygienic advice and voice therapy ${ }^{(2)}$.

Ponosurgery is a laryngeal surgery done solely or primarily to improve phonation which requires some glottic adjustment by altering the physical characteristics, placement, contour and function of the vocal folds. Conventional microlaryngeal phonosurgery (cold instrument technique) requires small, sharp and precise instruments ${ }^{(3)}$.

More knowledge about mechanism of change of voice is required to be known by the phonosurgeon before any microlaryngeal phonosurgery is preferred e.g. vocal fold lesions that impair vibratory movements require excision (vocal fold polyp), incision and suction (Reinke's space edema) ${ }^{(4)}$.

The goal of the phonosurgical procedures is to restore the integrity of the vocal organ (restoring the functional phonatory glottis) while preserving the respiratory function by achieving:

1. Smooth edge of the V.Fs. with a freely mobile mucosa.

2. Optimal coaptation of the glottis.

3. Abolishing phonatory glottal gap ${ }^{(5)}$.

Voice use after the microlaryngeal phonosurgery is step by step during the healing phase. After the first week of voice rest, voice may be still dysphonic but acceptable for a limited use ${ }^{(\mathbf{1})}$.

Vocal rehabilitation following phonosurgery includes two processes which are healing of the wound caused by surgery and vocal function recovery.
Voice training enables patients to establish or re-establish the physiological balance among the vocal organs and to correct the erroneous conditioned reflex caused by the vocal fold lesions to enable restoration of normal phonation function through changing the vocal environment and condition of the patients ${ }^{(6)}$.

Voice therapy is an integral component of intervention and contributes to both its efficacy and effectiveness that includes: identification of phonotraumatic behaviors which may have contributed to the original problem, vocal health and hygiene counseling, direct exercises to re-strengthen and re-balance the laryngeal mechanism to produce optimal voice ${ }^{(7) \text {. }}$

The accent method of voice therapy is often successful but requires active participation from the patient which turns off the patient's consciousness from the inclosed phonatory mechanism and strikes a balance between subglottic pressure and glottal activity $^{(8)}$.

\section{AIM OF THE STUDY:}

The aim of the accent method of voice therapy after microlaryngeal phonosurgery is to encourage "good vibrations" of the vocal folds. This involves creating and maintaining a healthy sound by repeating exercise regularly in order to reduce vocal fold swelling (oedema), prevent any potential vocal fold scarring and promote efficient voice production in the future ${ }^{(9)}$.

\section{PATIENTS AND METHODS:}

\section{Sampling:-}

- Target population: patients with dysphonia due to "minimal associated pathological lesions" (MAPLs) prepared for microlaryngeal phonosurgery.

- Type (method) of sampling: nonprobability purposive sample. 
- Sample size: 100 patients, 50 adult patients of both sexes received the "accent exercises" of voice therapy 1week after microlaryngeal phonosurgery as a study group (G1). In addition, 50 other adult patients of both sexes as a control group (G2) received only voice hygienic advice (due either inability to attend regular therapy sessions for remote residence or dropped out cases whose attendance was irregular and patients on waiting list).

All the patients were recruited from the outpatient clinic of the Phoniatrics Unit, Ain-Shams University Hospitals who fulfilled the inclusion criteria and agreed to participate in the study, then the phonosurgery was done. Also, the course of the "accent exercises" of voice therapy was given only to (G1). The study was carried out between November 2017 and January 2019.

\section{Inclusion criteria:-}

- Patients prepared for cold instrument microlaryngeal phonosurgery.

- Receiving voice hygienic advice before and after the surgery, both the study (G1) and control (G2) groups.
- Started the "accent exercises" of voice therapy 1week after the surgery, only the study group (G1).

\section{Exclusion criteria:-}

- Patients following laser microlaryngeal pnonosurgery.

- Those on any regular medication (anticholinergics, antihistaminics, diuretics, androgens, contraceptive pills with high progesterone and steroid inhalers) which might have an effect on voice quality.

\section{Study design:-}

The participants were randomly categorized into two groups. Group 1 (G1) comprised of 50 patients (29 males and 21 females) with age ranging from 20 to 65 years $($ mean $=43.1)$. This group received voice hygiene advice plus the "accent exercises" of voice therapy (study group). Group 2 (G2) comprised of 50 patients (28 males and 22 females) with age ranging from 22 to 70 years $($ mean $=42.5)$. This group was given voice hygiene advice only (control group).

It was a comparative study between two groups who presented with a variety of benign vocal fold lesions showing in the following table.

Table: The variety of the minimal associated pathological lesions included in the study.

\begin{tabular}{|l|c|c|}
\hline & $\begin{array}{c}\text { G1 } \\
\text { (Study group) }\end{array}$ & $\begin{array}{c}\text { G2 } \\
\text { (Control group) }\end{array}$ \\
\hline Polyp & 21 & 21 \\
\hline Cyst & 14 & 14 \\
\hline Reinke's space edema & 8 & 7 \\
\hline Polypoid degeneration & 4 & 4 \\
\hline Nodules & 3 & 4 \\
\hline Total & 50 & 50 \\
\hline
\end{tabular}

\section{RESULTS}

\section{Auditory perceptual assessment (APA):-}

For the second assessment after the microlaryngeal phonosurgery:

Auditory perceptual assessment items: overall grade (G), strained (S), leaky (L), irregular (I), pitch, loudness and associated laryngeal functions showed that no statistically significant differences in improvement between both groups (diagram $1)$. 


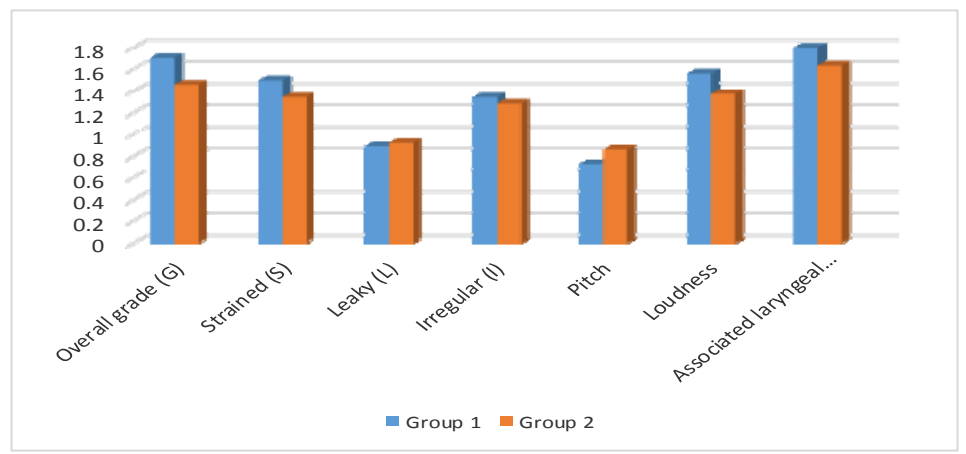

Diagram 1: Histogram representation of the mean difference in the improvement of APA between study group (G1) and control group (G2) after the $2^{\text {nd. }}$ assessment (microlaryngeal phonosurgery to both groups).

\section{For the third assessment:}

Auditory perceptual assessment items showed that statistically difference in improvement of items overall grade $(\mathrm{G})$, strained (S), leaky (L), pitch, loudness and associated laryngeal functions between both groups indicated that study group (G1) has improved with a high degree of significance as compared to control group (G2). The difference in improvement in item irregular (I) was not significant (diagram 2).

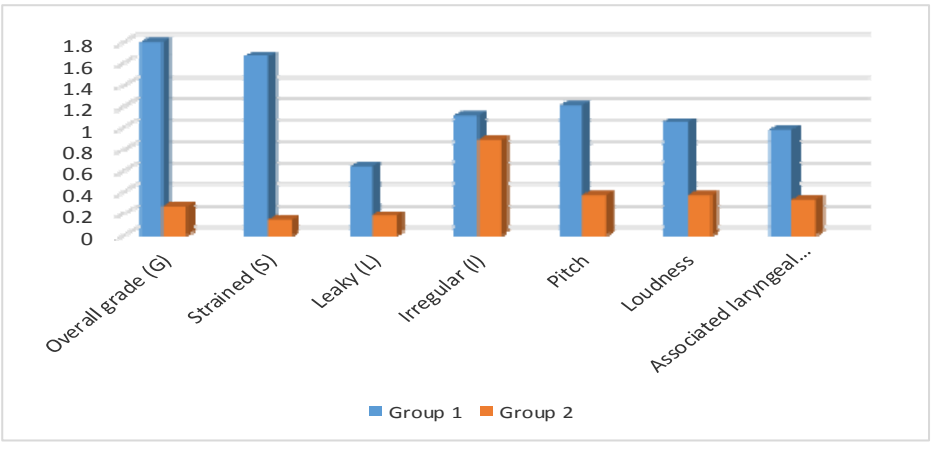

Diagram 2: Histogram representation of the mean difference in the improvement of APA between study group (G1) and control group (G2) after the $3^{\text {rd. }}$ assessment (accent exercises given to G1 only and voice hygienic advice to both groups).

\section{High-speed Video-kymography}

\section{parameters:-}

For the second assessment after the microlaryngeal phonosurgery:
There were no statistically significant differences regarding all high-speed videokymography parameters between study group (G1) and control group (G2) (diagram $3)$.

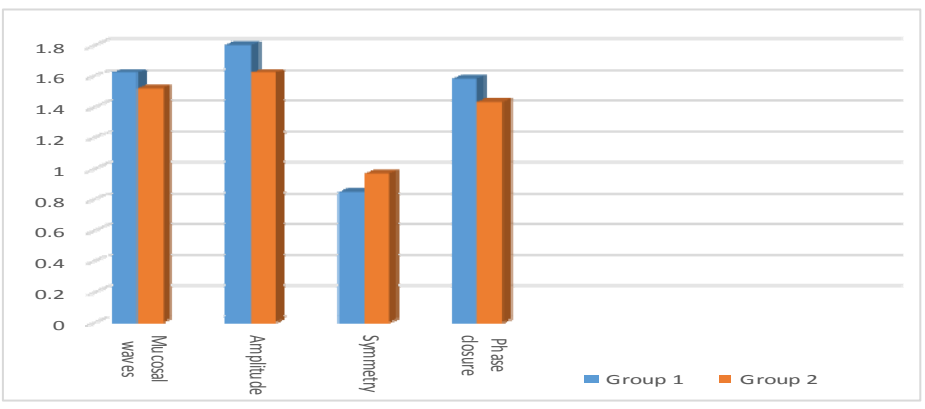

Diagram 3: Histogram representation of the mean difference in the improvement of high-speed videokymography parameters between study group (G1) and control group (G2) after the $2^{\text {nd. }}$ assessment (microlaryngeal phonosurgery to both groups). 


\section{For the third assessment:}

There were differences between both groups. Patients in (G1) receiving the accent exercises showed improvement in mucosal mucosal waves, amplitude, symmetry and phase closure with a high degree of statistically significant. While patients in (G2) received only counseling regarding voice hygienic advice showed no significant improvement changes for all observations (diagram 4).

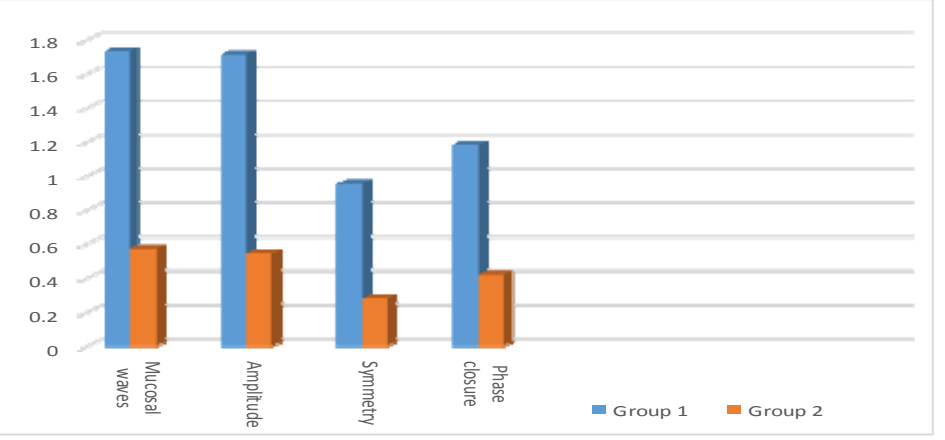

Diagram 4: Histogram representation of the mean difference in the improvement of the high-speed videokymography parameters between study group (G1) and control group (G2) after the $3^{\text {rd. }}$ assessment (accent exercises given to G1 only and voice hygienic advice to both groups).

\section{Acoustic analysis:}

For the second assessment after the microlaryngeal phonosurgery:
There were no statistically significant differences regarding all the acoustic analysis parameters between both groups (diagrams 5, 6).

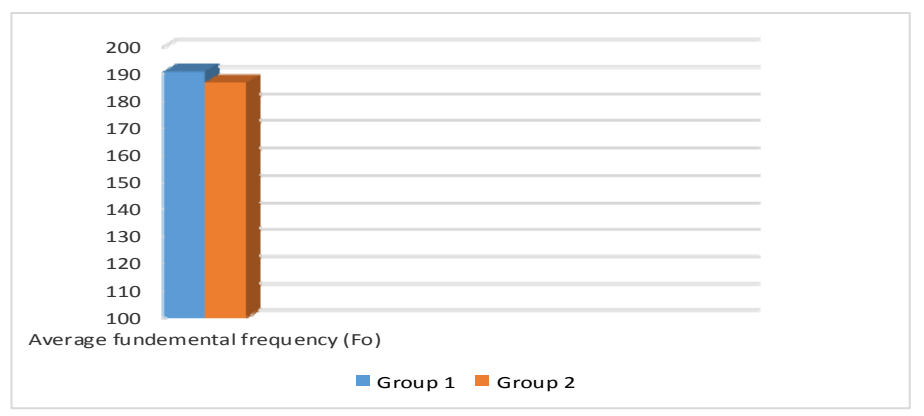

Diagram 5: Histogram representation of the mean difference in improvement of Average fundamental frequency (Fo) between study group (G1) and control group (G2) after the 2nd. assessment (microlaryngeal phonosurgery to both groups).

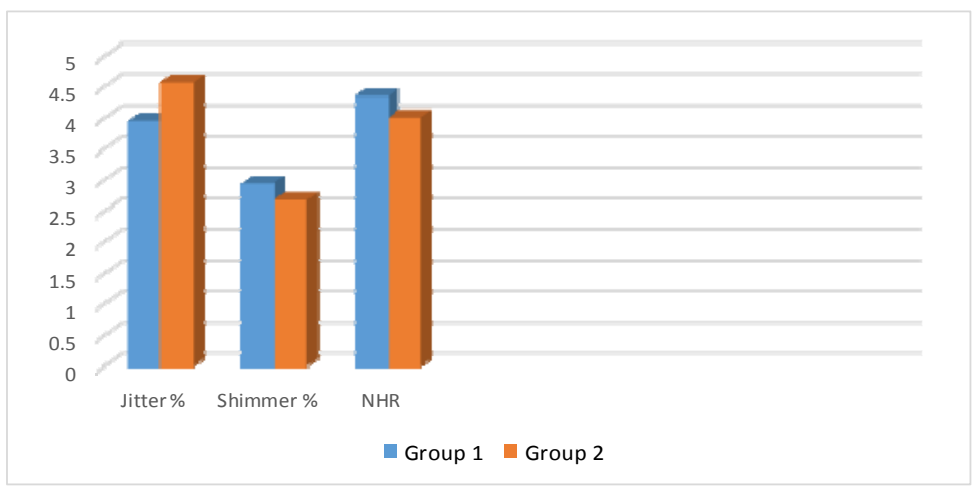

Diagram 6: Histogram representation of the mean difference in improvement of Jitter \%, Shimmer \% and NHR between study group (G1) and control group (G2) after the 2nd. assessment (microlaryngeal phonosurgery to both groups). 
For the third assessment:

The difference in improvement between the study group (G1) received the accent exercises and the control group (G2) received only voice hygienic advice was statistically significant in average fundamental frequency (Fo), Jitter \% and Shimmer \% while, it was statistically highly significant in Noise to Harmonic Ratio (NHR) (diagrams 7,8).

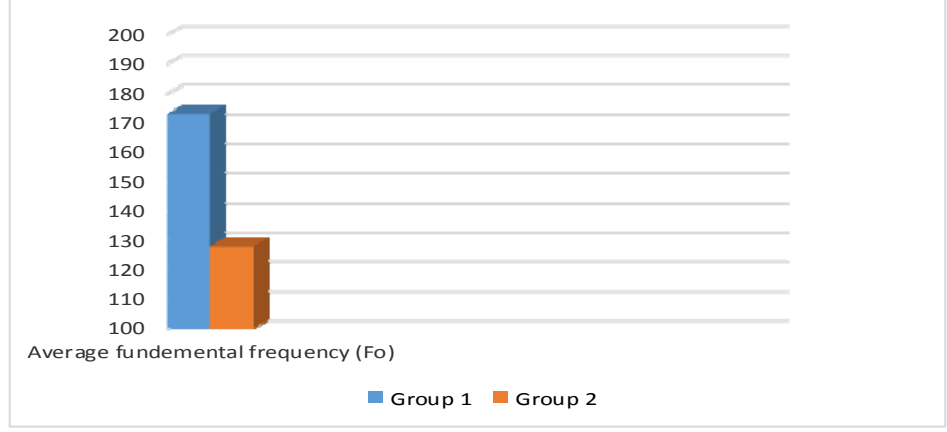

Diagram 7: Histogram representation of the mean difference in improvement of Average fundamental frequency (Fo) between study group (G1) and control group (G2) after the $3^{\text {rd. }}$ assessment (accent exercises given to G1 only and voice hygienic advice to both groups).

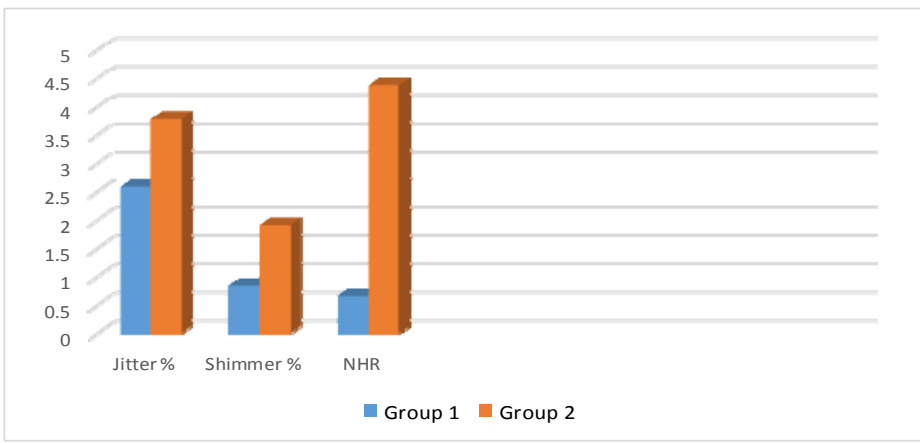

Diagram 8: Histogram representation of the mean difference in improvement of Jitter $\%$, Shimmer $\%$ and NHR between study group (G1) and control group (G2) after the $3^{\text {rd. }}$ assessment (accent exercises given to G1 only and voice hygienic advice to both groups).

\section{Aerodynamic measures:-}

For the second assessment after the microlaryngeal phonosurgery:
There were no statistically significant differences regarding all the aerodynamic measures between study group (G1) and control group (G2) (diagrams $9,10,11,12)$.

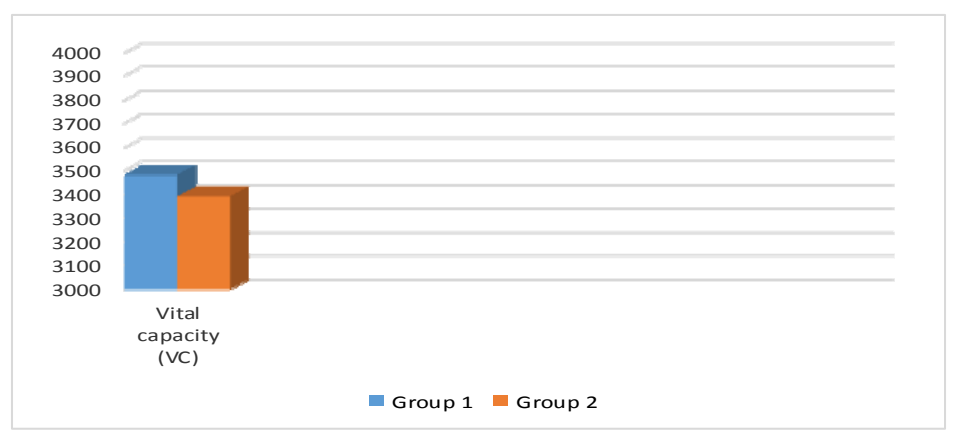

Diagram 9: Histogram representation of the mean difference in the improvement of vital capacity (VC) between study group (G1) and control group (G2) after the 2nd. assessment (microlaryngeal phonosurgery to both groups). 


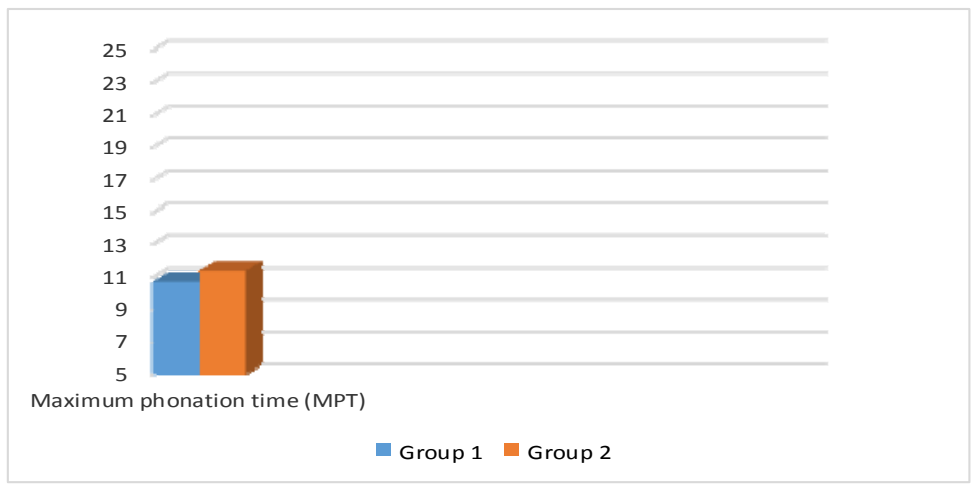

Diagram 10: Histogram representation of the mean difference in the improvement of maximum phonation time (MPT) between study group (G1) and control group (G2) after the 2nd. assessment (microlaryngeal phonosurgery to both groups).

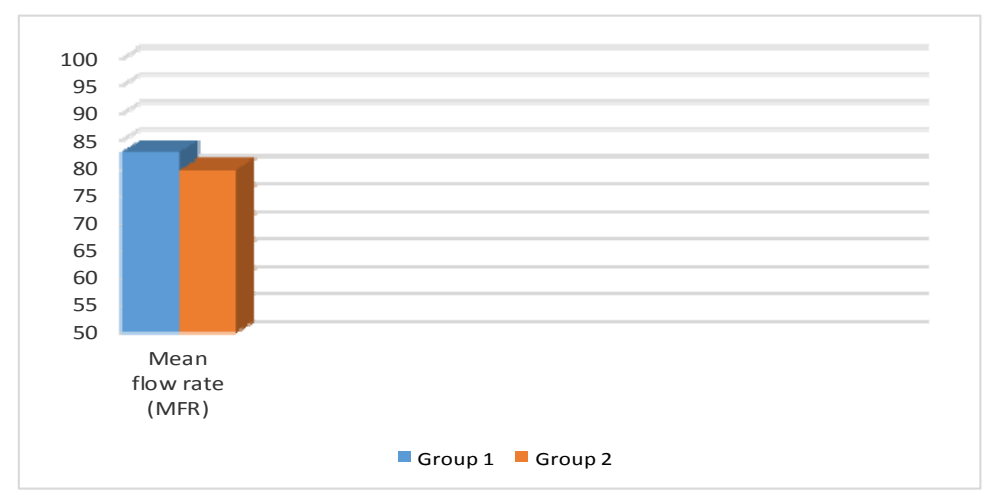

Diagram 11: Histogram representation of the mean difference in the improvement of mean flow rate (MFR) between study group (G1) and control group (G2) after the 2nd. assessment (microlaryngeal phonosurgery to both groups).

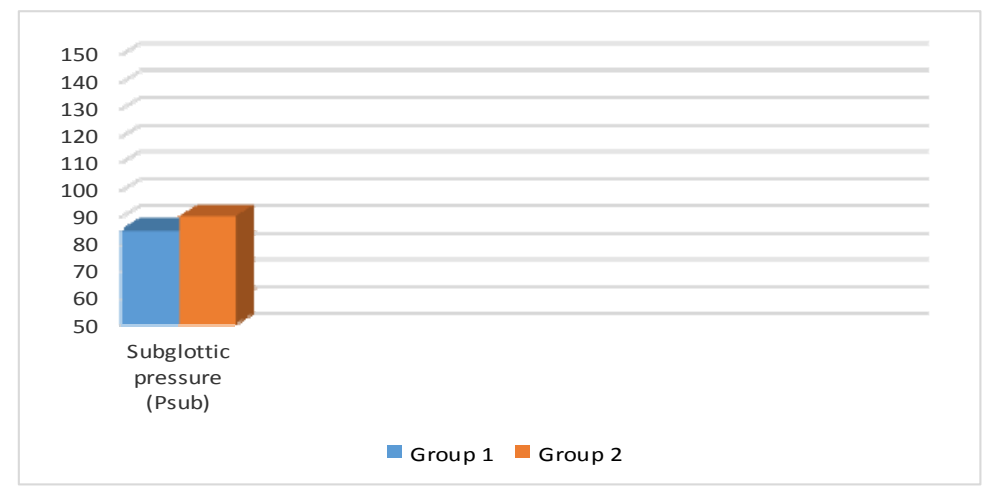

Diagram 12: Histogram representation of the mean difference in the improvement of subglottic pressure (Psub) between study group (G1) and control group (G2) after the 2nd. assessment (microlaryngeal phonosurgery to both groups).

\section{For the third assessment:}

The difference in improvement between both groups was statistically significant in vital capacity (VC) and mean flow rate
(MFR) while it was statistically highly significant in favor of (G1) in maximum phonation time (MPT) and subglottic pressure (Psub) (diagramss 13, 14, 15, 16). 
Samia El-Sayed Bassiouny, et al.,

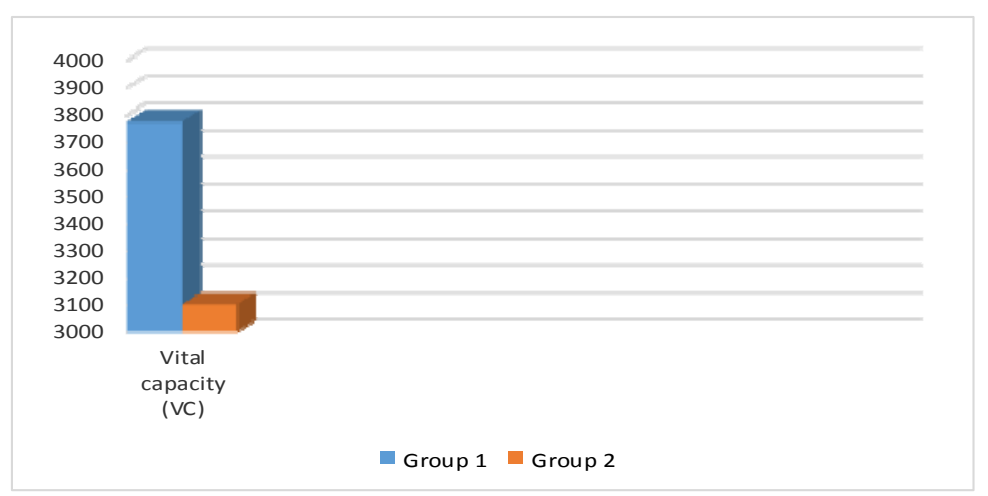

Diagram 13: Histogram representation of the mean difference in the improvement of vital capacity (VC) between study group (G1) and control group (G2) after the $3^{\text {rd. }}$ assessment (accent exercises given to G1 only and voice hygienic advice to both groups).

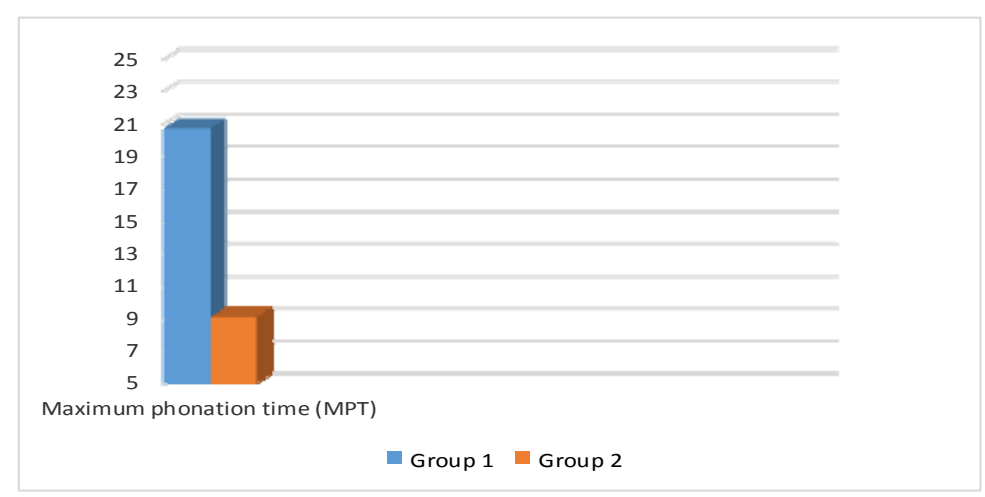

Diagram 14: Histogram representation of the mean difference in the improvement of maximum phonation time (MPT) between study group (G1) and control group (G2) after the $3^{\text {rd. }}$ assessment (accent exercises given to G1 only and voice hygienic advice to both groups).

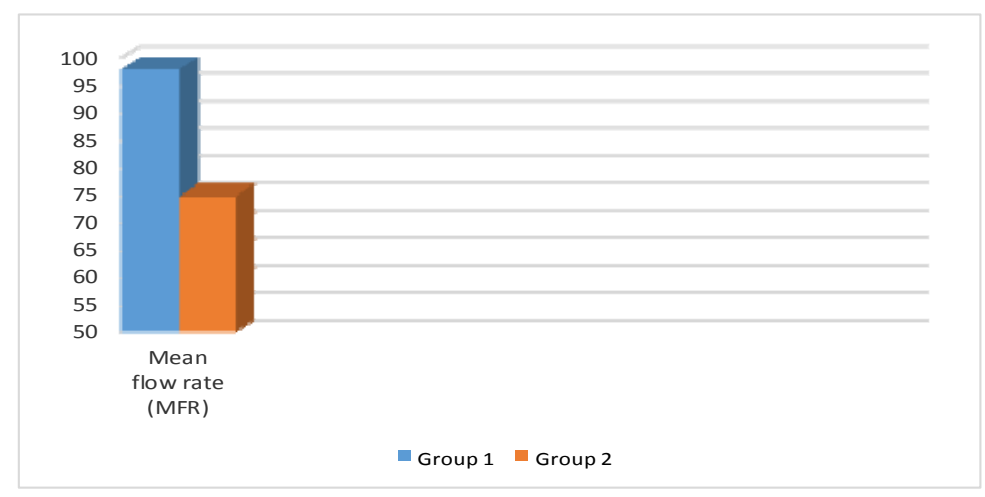

Diagram 15: Histogram representation of the mean difference in the improvement of mean flow rate (MFR) between study group (G1) and control group (G2) after the $3^{\text {rd. }}$ assessment (accent exercises given to G1 only and voice hygienic advice to both groups). 


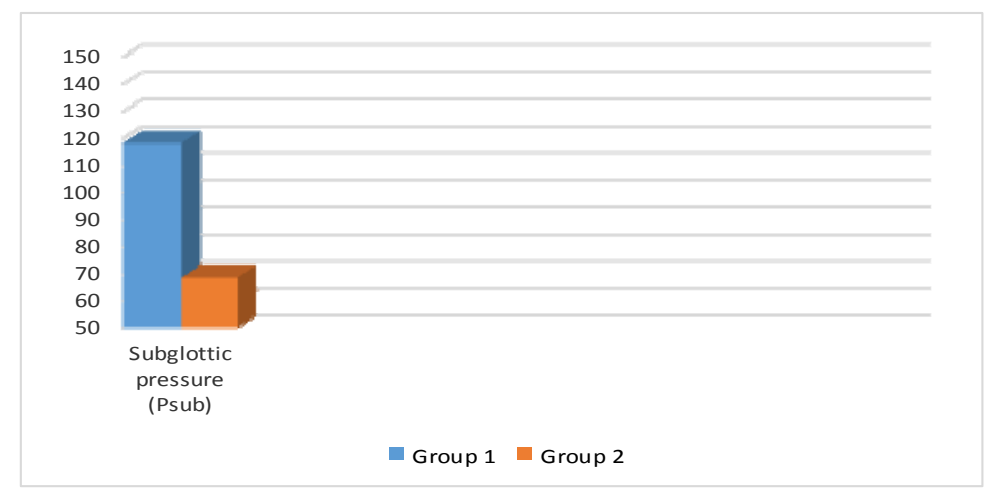

Diagram 16: Histogram representation of the mean difference in the improvement of subglottic pressure (Psub) between study group (G1) and control group (G2) after the $3^{\text {rd. }}$ assessment (accent exercises given to G1 only and voice hygienic advice to both groups).

\section{DISCUSSION:}

This study based on the concept of group comparisons. The role of the "accent exercises" of voice therapy method were tested by assessment of the effects of accent exercises given to study group (G1) for correction of the faulty vocal techniques (habits) against the effect of voice hygiene advice given to both study (G1) and control (G2) groups. By separating the two elements of behavior readjustment therapy, one may be able to demonstrate the role of each.

The therapeutic mode of action of the accent exercises after doing microlaryngeal phonosyrgery rest on the improved expiratory air support and timing in relation to the onset of phonation ${ }^{(9)}$. This in turn, improve glottal aerodynamics with increased air flow leading to restoration of the symmetry, increase of the amplitude of the glottal wave and decrease of any phonatory gap.

Several studies attempted to study the efficacy of the accent method of voice therapy for benign lesions of vocal folds and some organic voice disorders $(10,6,11 \& 12)$. Other studies evaluated the effect of the accent method of voice therapy as a holistic approach (both voice hygienic advice and accent exercises together) in patients with symptoms of the change of voice after the microlaryngeal phonosurgery ${ }^{(3,13 \& 14)}$ and in comparison to normal subjects ${ }^{(2,15 \& 16)}$.

These previous studies used acoustic and aerodynamic measures yielded inconclusive and contradictory results, probably due to the small number of subjects included.

In this study, the "accent exercises" were given in a setup that may establish a cause-and-effect relation between the tested technique implemented by the phoniatrician and the changes in the patient's vocal behavior as well as other investigated parameters. Both the acoustic analysis and the aerodynamic measures can provide means to monitor changes (follow up cases) resulting from treatment especially after the microlaryngeal phonosurgery of benign V.F. lesions and after applying the course of "accent exercises" of voice therapy.

This study revealed that the "accent exercises" of voice therapy has a big role in some selected cases after microlaryngeal phonosurgery, which have inclusion criteria as the following:

Big sized lesion.

Reaction on the opposite side.

Long-term lesion.

Compensatory reactions like ventricular bands increased either in size (red 


\section{Samia El-Sayed Bassiouny, et al.,}

inflamed edematous) or in more adduction sharing in phonation (hyperadduction).

$>$ Associated non-organic elements like hyperfunctional or hypofunctional element.

$>$ Combined lesions e.g. V.F. polyp on top of Reinke's edema and subepithelial hemorrhage with V.F. polyp or cyst.

$>$ Technical problems that may appear during surgery.

The general trend of the final results (after third assessment) showed a significant difference in improvement of vocal function parameters in favor of the "accent exercises" in (G1).

Regarding APA in this study, all the participants after microlaryngeal phonosurgery, the improvement of items strained (S) and leaky (L) in the subgroups of vocal fold polyp and cyst as well as improvement of item irregular (I) in the subgroups of vocal fold reinke's edema and polypoid degeneration was mild quality of improvement because the lesion was only removed while patients sill practicing faulty vocal behavior techniques resulting in dysphonia.

In $\mathrm{G} 1$, after the course of the "accent exercises", the improvement of these bad quality of voice strained (S) and leaky (L) in the subgroups of vocal fold polyp and cyst was highly significant as well as the improvement in item irregular (I) in the subgroups of vocal fold reinke's edema and polypoid degeneration was highly significant due to the effect of increased air flow in response to the accent exercises on the sound pressure level (SPL) and fundamental frequency $(\mathrm{Fo})$.

Other studies revealed that the perceptual assessment of voice pathology was statistically significant improved to normal pitch, loudness and quality after endolaryngeal phonomicrosurgery $\left.{ }^{(13 \& 17}\right)$.
In the high-speed video-kymography findings, there were statistically significant difference in improvement of mucosal waves, amplitude and symmetry of both V.Fs. in favor of (G1) indicating the effect of the "accent exercises" in controlling these morphological deficits in vocal pathology. This favorable effect of the accent exercises in (G1) was confirmed by the statistically significant positive changes observed only in (G1) that were normal mucosal waves, amplitude and phase closure with periodicity for each vocal fold and symmetrical both vocal folds, which latter may reflect more changes in the glottal behavior.

Several studies used high-speed imaging video-kymography demonstrating the effect of phonosurgery for benign vocal fold lesions was documented statistically significant improvement in the mucosal waves, amplitude and phase closure ${ }^{(3,13 \& 18)}$.

Our study observed that there has been some improvement regarding phase closure in both groups (G1 and G2), reflecting the role of expert phonosurgeon during the microlaryngeal phonosurgery indicating precise dealing with the lesion and good post-operative follow up aiming to proper tissue healing.

As regard the acoustic analysis, there was evidence for the difference in improvement of all parameters inbetween the groups (G1 and G2) in favor of (G1) because of the rule of the rhythmic accentuated accent exercises on sound pressure level (SPL) to increase airflow rate and produce variable degree of increase in fundamental frequency (Fo) and decrease in Jitter \%, Shimmer \% and Noise to Harmonic Ratio(NHR).

With improvement in Jitter \%, Shimmer $\%$ and NHR documented also in many studies following voice therapy for some benign V.F. lesions ${ }^{(19 \& 7)}$ and after microlaryngeal phonosurgery ${ }^{(3 \& 15)}$, the fundamental frequency (Fo) revealed remarkable 
improvement with increase in pitch bringing back patient's own vocal pitch. It is probably due to the tissue load (mass effect) that is important to lower the fundamental frequency (Fo) value before the surgical removal of the benign V.F. lesions ${ }^{(15)}$. In contrast, other studies have observed decreased values of the fundamental frequency (Fo) following microlayngeal phonosurgery to benign V.F. lesions $\left.{ }^{(13} \& 14\right)$.

Statistically highly significant reduced values of Jitter \% and Shimmer \% after voice therapy for the benign V.F. lesions also was found ${ }^{(10)}$. In contrast to this study, only a statistically significant decrease in Shimmer \% but not in Jitter \% following the microlayngeal phonosurgery was found ${ }^{(3)}$. The significant decrease in Jitter \% was reliable indicator than Shimmer \% when assessing the benign V.F. lesions has been obtained after the microlayngeal phonosurgery ${ }^{(2)}$.

All these contradictions while assessing the perturbation attributed to methodological dissimilarity among the studies and application of different analytical systems and software for signal processing.

The significant difference in improvement of the aerodynamic measures in favor of (G1) was selective. The improvement of these measures in different subgroups of benign lesions of the vocal folds was already detectable in the $2^{\text {nd }}$ assessment measures (post-surgery) but, not statistically significant was detected. All the improved items continued in the $3^{\text {rd }}$ assessment measures (post accent exercises of voice therapy) with significant/highly significant improvement.

These further improvements in aerodynamic measures, which observed in this study, because the accent exercises train the patient's abdominal breathing movements (by alternating contraction and release) and open throat postures to produce easy voicing (improved expiratory air support and timing in relation to the onset of phonation). Learning of new phonatory behaviors (accent exercises) will enhance the Bernoulli's effect to restore the glottic wave with better adjustment and symmetry of the vibrator (vocal fold mucosa) striking a balance between subglottic pressure and glottal activity and seeks to optimize flexibility of voice generation.

In this respect, our findings supported the earlier studies ${ }^{(13 \& 20)}$ have showed increase MFR and MPT after microlayngeal phonosurgery to benign V.F. lesions.

The highly significant improvement of maximum phonation time (MPT), mean flow rate (MFR) and subglottic pressure (Psub) reflects an improvement in phase closure noticed in the high-speed video-kymography findings.

\section{Conclusion:}

The basic principle of microlaryngeal phonosurgery is to maintain or improve the functional structure of the vocal folds by respecting its layered structure. This is achieved by means of minimal tissue excision, minimal disruption of the superficial layer of the lamina propria and preservation of the epithelium especially at the vibrating edge.

Patients following the microlaryngeal phonosurgery may still have variable degree of dysphonia because phonosurgery does not improve faulty vocal techniques and patients still practice bad vocal habits of abuse and misuse of voice which is more injurious to the delicate vocal fold structures.

As the minimal associated pathological lesions (MAPLs) of the vocal folds is a result of phonotrauma (abuse and misuse of voice). Voice use after microlaryngeal phonosurgery is step-by-step during the healing phase. After the first week of voice rest, voice may be still dysphonic but acceptable for a limited use. 
Having the course of "Accent Method" of voice therapy after doing microlaryngeal phonosurgery is almost always a good idea in order to make the person aware of circumstances and habits of voice use that may have led to recurrence of the lesion in the first place.

Voice training enables patients to establish or re-establish the physiological balance among the vocal organs, in order to correct the erroneous conditioned reflex caused by the vocal fold lesions to enable restoration of normal phonation function through changing the vocal environment and condition of the patients.

\section{REFERENCES:}

1. American Speech-Language Hearing Association (ASHA) (2016): Knowledge and skills for speech-language pathologists with respect to Behavioral readjustment therapy (the Accent Method). Retrieved from www.asha.org/policy.

2. Li Lin; Na Sun; Qiuhua Yang; Ya Zhang; Ji Shen; Lixin Shi; Qin Fang and Guangbin Sun (2014): Effect of voice training in the voice rehabilitation of patients with vocal cord polyps after surgery. Experimental and therapeutic medicine. Laryngoscope, 286: $52-65$.

3. Zeitels, S.M.; Hillman, R.E.; James, A. and Burns, E. (2012): Phonomicrosurgery in singers and performing artists: treatment outcomes, management theories and future directions. Ann. Otol. Rhinol. Laryngology, 111: 21-40.

4. Abitbol, J. (2012): Surgical management of non-neoplstic vocal fold lesions: laser versus cold knife excision. Current Opinion in Otolaryngology, Head and Neck Surgery, 18: 514-523.

5. Kotby, M.N. (1995): The accent method of voice therapy. Singular Publishing Group, Inc., San Diego, California.

6. Gartner-Schmidt, J.; Roth, D.; Zullo, T. and Rosen, C. (2013): Quantifying component parts of indirect and direct voice therapy related to different voice disorders. Journal of Voice. 27: 210-216.

7. Behrman, A.; Rutledge, J.; Hembree, A. and Sheridan, S. (2014): Vocal hygiene education, voice production therapy and the role of patient adherence. Journal of speech, language and hearing research, 51: 350-366.

8. Kotby, M.N.; El-Sady, S.R.; Bassiouny, S.E.; Abou Rass, Y.A. and Hegazi, M.A. (1991): Efficacy of the Accent Method of voice therapy. Journal of voice, 5: 316.

9. Berry, D.; Verdolini, K.; Chan, R. and Titze, I. (2014): Indications of an optimum glottal width in vocal production. Journal of Speech and Hearing Research. 120: 407413.

10. Bassiouny, S. (1998): Efficacy of the accent method of voice therapy. Folia Phoniatrica et Logopedica, 50: 146-164.

11. Benninger, M.S. (2007): Management of limited vocal fold benign lesions: a prospective randomized trial. Laryngoscope, 180: 1-17.

12. Holmberg, E.B.; Hillman, R.F.; Hammaberg, B.; Sodersten, M. and Doyle, P. (2011): Efficacy of behaviorally based voice therapy protocol for vocal fold nodules. Journal of Voice. 45(3): 395-412.

13. Katusic, S.S.; Horga, D. and Zrinski, K.V. (2008): Longitudinal study of voice before and after phonosurgery for removal of a polyp. Clinical linguistics and phonetics, 22: $57-63$.

14. Petrovic-Lazic, M.; Babac, S. and Vukovic, M. (2010): Acoustic analysis and aerodynamics of patients with vocal fold polyps. Journal of Voice, 23: 84-91.

15. Murry, T. (2001): Pre and post-operative phonotherapy. Otolaryngologic Clinics of North America. Journal of Sing 57: 39-42.

16. Behlau, M. (2007): Voice therapy and microsurgery of the larynx. Proceeding of $10^{\text {th }}$. International Work Shop on Laser Voice Surgery and Voice Care. Paris, France.

17. Uloza, V.; Viktoras, S. and Ingrida U. (2005): Perceptual and acoustic assessment 
of voice pathology and efficacy of endolaryngeal phonomicrosurgery. Journal of Voice; 19:138-145.

18. Larsson, H.; Hertegard, S. and Lindestad, P. (2009): Vocal fold vibrations: high-speed imaging, kymography and acoustic analysis. Prospective study for polyps, cysts and treatment. The Laryngoscope. 110(12): 117122.
19. Kotby, M.N.; Shiromoto, O. and Hirano, M. (1993): The Accent Method of voice therapy: Effect of accentuations on Fo, SPL and air flow. Journal of voice, 7: 319.

20. Geovani A.; Revis J. and Triglia J.M. (1996): Objective aerodynamic and acoustic measurement of voice improvement after phonomicrosurgery. Laryngoscope 109: 656-660.

\section{تقييم دور العلاج باستخدام تدريبات الصوت بطريقة "سميث أكسنت" بعد الجراحات الصوتية الدقيقة فى الحنجرة

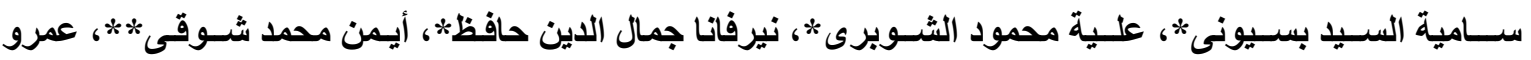

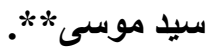

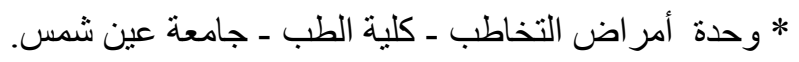 \\ *** وحدة أمر اض التخاطب ـ الأكاديمية الطبية العسكرية.}

المقدمة:

تعتبر البحة الصوتية هى مصطلح عام للتعبير عن أعراض الأمر اض الته التى تصيب الحنجرة، فهى تؤثر بشكل كبير

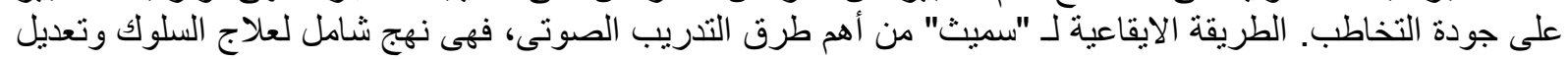

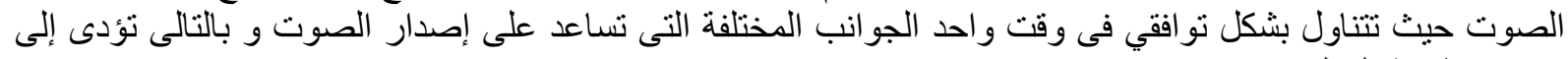
تحسنه بطبيعة الحال. ت تلترل

الهُف من البحث:

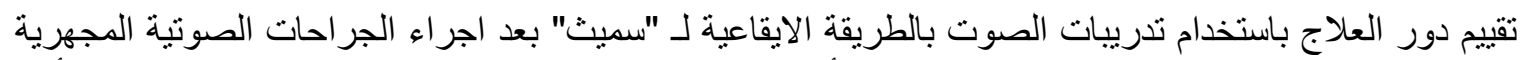

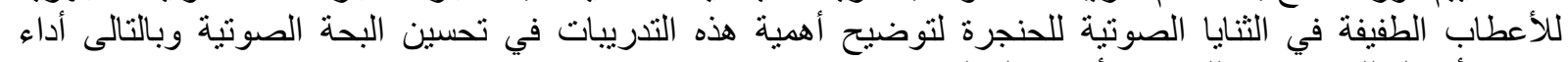

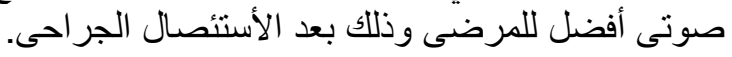
المرضى و طرق البحث:

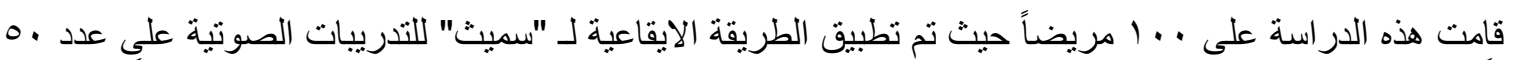

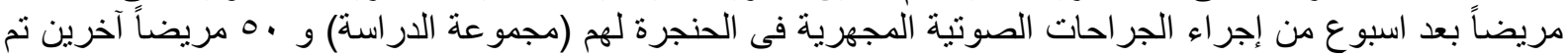

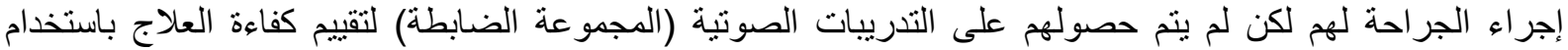

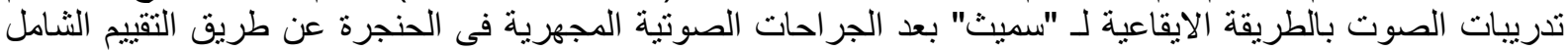

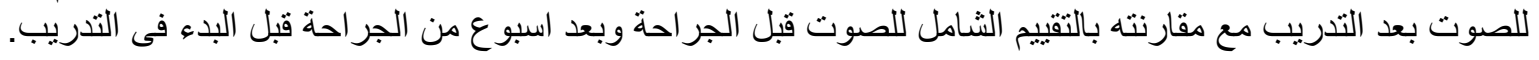

النتائج:

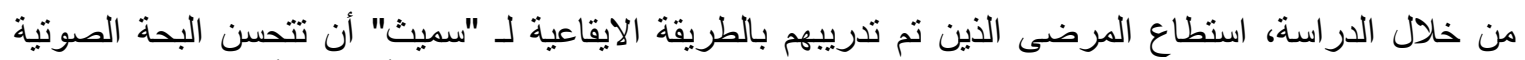

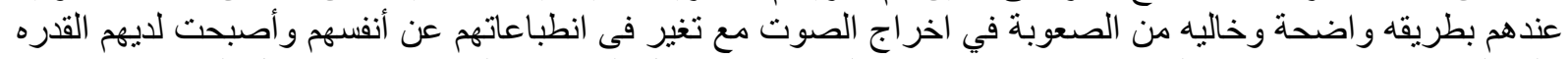

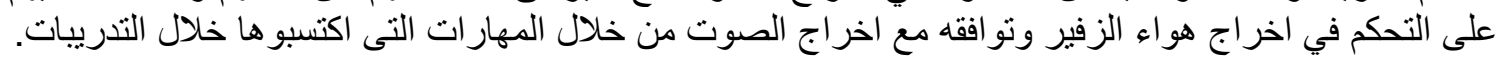

الخاتمة:

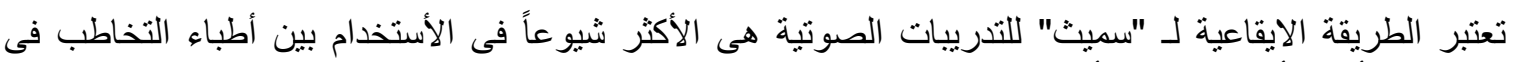

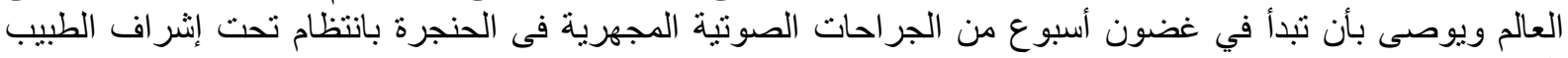

